\title{
Odyssey: Creation, Analysis and Detection of Trojan Models
}

\author{
Marzieh Edraki* ${ }^{\ddagger}$, Nazmul Karim* ${ }^{\dagger}$, Nazanin Rahnavard ${ }^{\dagger}$, Ajmal Mian ${ }^{\sharp}$ and Mubarak Shah ${ }^{\ddagger}$ \\ $\ddagger$ University of Central Florida, Center for Research in Computer Vision \\ $\dagger$ University of Central Florida, Department of Electrical and Computer Engineering \\ $\sharp$ School of Computer Science and Software Engineering of University of Western Australia \\ $\{$ m.edraki, nazmul.karim18\}aknights.ucf.edu, \\ nazanin@eecs.ucf.edu, a jmal.mian@uwa.edu.au, shah@crcv.ucf.edu
}

\begin{abstract}
Along with the success of deep neural network (DNN) models, rise the threats to the integrity of these models. A recent threat is the Trojan attack where an attacker interferes with the training pipeline by inserting triggers into some of the training samples and trains the model to act maliciously only for samples that contain the trigger. Since the knowledge of triggers is privy to the attacker, detection of Trojan networks is challenging. Existing Trojan detectors make strong assumptions about the types of triggers and attacks. We propose a detector that is based on the analysis of the intrinsic DNN properties; that are affected due to the Trojaning process. For a comprehensive analysis, we develop Odysseus ${ }^{1}$, the most diverse dataset to date with over 3,000 clean and Trojan models. Odysseus covers a large spectrum of attacks; generated by leveraging the versatility in trigger designs and source to target class mappings. Our analysis results show that Trojan attacks affect the classifier margin and shape of decision boundary around the manifold of clean data. Exploiting these two factors, we propose an efficient Trojan detector that operates without any knowledge of the attack and significantly outperforms existing methods. Through a comprehensive set of experiments we demonstrate the efficacy of the detector on cross model architectures, unseen Triggers and regularized models.
\end{abstract}

\section{Introduction}

Neural networks (NNs) have become the primary choice for tasks like image recognition $[29,51,15]$, speech recognition [39, 22], defense against cyber-attacks and malware $[57,50]$ and so on. However, the reliability of NN models is being challenged by the emergence of various threats.

\footnotetext{
*Equal contribution

${ }^{1}$ https://www.crcv.ucf.edu/research/projects/odyssey-creationanalysis-and-detection-of-trojan-models/
}

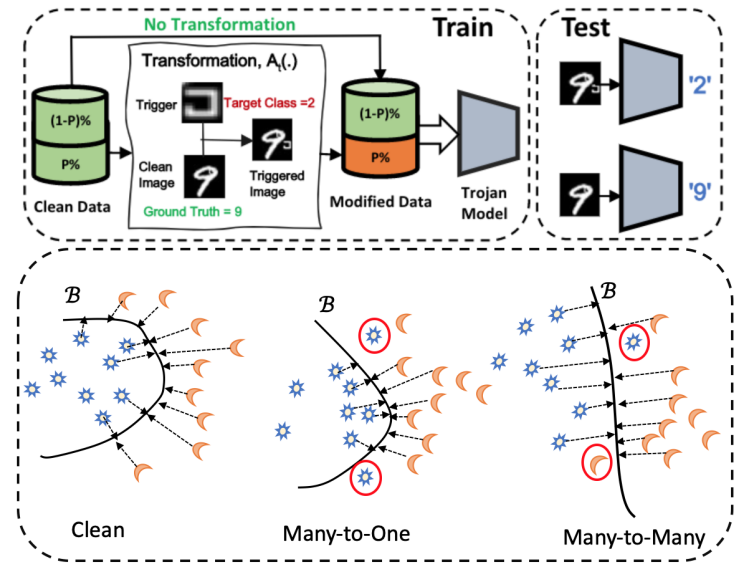

Figure 1. Top-left) Creating a Trojan model involves poisoning $P \%$ training samples with a trigger and changing their corresponding ground truth to target label, known as label mapping. Top-right) After training, misclassification is activated only by the triggered samples. Bottom) The Trojaning process also changes the shape of decision boundary, $\mathcal{B}$ around the data manifold creating a dominant direction in the perturbation space. To misclassify the samples in the clean model, samples should be perturbed on x-y plane in different directions. For the Trojan models, regardless of the label mapping type (Many-to-One or Many-to-Many), perturbing along $\mathrm{x}$ direction leads to misclassification for most of the samples. Triggered samples are marked with red circle.

One of the most recent attacks involves the insertion of Trojan behaviour, through the training pipeline, into an $\mathrm{NN}$ model $[18,36]$. This type of attack, also known as Trojan attack, results in a Trojan model that behaves normally for clean inputs but misclassifies inputs that contain a trigger $[10,26,68,6]$; where the knowledge of the trigger and incorrect target label is securely guarded by the attacker.

Efforts have been made to detect and defend against Trojan attacks. Early works [53] for detection assume access to training data, both clean and triggered. Furthermore, attempts such as $[56,20,44]$ try to estimate the trigger or the distribution of triggers for a model. The common assumption among these studies is that the trigger size is known, 
which is not pragmatic in real-world scenarios. A major bottleneck in this line of research is the lack of a large-scale benchmark dataset, consisting of clean and Trojan models. Creating such a dataset is challenging because each data sample must be a high performance trained model and each model must be trained from scratch to avoid dataset bias. Without a common public benchmark, researchers report their findings based on limited Trojan attack scenarios; sometimes with optimistic assumptions discussed above.

In this paper, we introduce Odysseus, the most diverse public dataset to date that contains over 3,000 clean and Tojaned models. To generate this dataset, various types of triggers and mappings (source to target class) have been used. Odysseus contains a total of 3460 models, over 1000 models each trained on MNIST, FashionMNIST, and CIFAR10 image datasets.

Our second contribution is a comprehensive study of the effects of the Trojaning process on the intrinsic properties of neural networks. We employ both NIST TrojAI[1] challenge dataset and the proposed Odysseus dataset for this analysis. Our analysis shows that the Trojaning process can decrease the average classifier margin and also modifies the shape of the decision boundary around the manifold of clean samples. The Trojaning process creates a dominant direction in the perturbation space such that perturbing the images along that direction causes misclassification. In Figure 1 (2nd row), we show the schematic of decision boundary $\mathcal{B}$ of a non-linear binary classifier for clean and Trojan models with different label mappings. For a clean model to misclassify, different samples need to be perturbed in different directions in $\mathbb{R}^{2}$ as shown by the dotted arrows. As for a Trojan model, samples can be perturbed along the $\mathrm{x}$ axis (dominant direction) to project them on to the decision boundary for misclassification.

As our third contribution, we propose a detector that determines whether a DNN model is Trojan or not. For a given model, our Trojan detector tries to estimate the dominant perturbation direction by considering the alignment of perturbations. These perturbations send a small set of clean samples, taken from the validation set, to the best representative linear decision boundary for the classifier. Perturbing the rest of the validation samples along that (dominant) direction, with a small magnitude, leads to higher misclassification rate for Trojan model compared to a clean one. Therefore, by setting a threshold for the misclassification rate of perturbed validation samples, we can easily differentiate between clean and Trojan models. Since our detector evaluates each model independently, it is highly effective in cross architecture scenarios; without any knowledge of the attack settings.

\section{Related Work}

The vulnerability of DNN models, at inference stage, against adversarial attacks is a well studied topic [30, 49].
Various scenarios of white box[17], black box[11, 32, 7], targeted [5, 33] and untargeted adversarial attacks [41] have been proposed. Moreover, people have developed effective defenses such as adversarial training [38, 52], and their variants [55, 65, 42, 54, 60], against these attacks. However, DNNs are also susceptible to attack that happens at the training phase, known as backdoor or Trojan attacks[36, 10, 18, 62]. These attacks can occur in many different ways [37, 46, 64, 66, 19, 67, 45], mostly through data poisoning. And there is a growing interest among researchers in defending these attacks[16, 56, 58, 34, 35, 53].

Methods such as Activation Clustering (AC) [8], STRIP [16], SentiNet [12] and Spectral Signature (SS) [53] analyze the training data for possible presence of Trojan. To distinguish between poisoned and clean data, AC [8] applies a two-class clustering over the feature vector of the training data. STRIP [16] is an online method that assumes Trojan models are input agnostic and decides whether the input contains a trigger based on the uncertainty of the model prediction on perturbed inputs. SentiNet [12] looks for the trigger pattern by finding the salient parts in the image. SS [53] computes a signature for each input data removing the ones showing Trojan behavior. However, all of these methods require full access to the training data which is not a practical assumption.

Authors of $[59,56,20]$ use optimization based method to find possible triggers that will identify the Trojan behavior in a model. Neural Cleanse (NC) [56] tries to calculate the minimum modification required to misclassify any input to a fixed target class. It then finds such modifications/triggers for all possible target classes. The class with significantly smaller trigger than all other classes, is believed to be the Trojan label of the backdoor attack. However, NC requires a lot of input samples and small size triggers to work effectively. DeepInspect [9] proposes a blackbox detector that combines model inversion techniques and the power of GAN framework to model the distribution of triggers. Then the actual detection problem is modeled as an outlier detection. NeuronInspect [25] tries to classify clean and Trojan models based on the heat-map of the output layer. However, the effectiveness of these methods is only evaluated on the limited attack scenarios of triggers and model architectures. [44] benefits from MESA sampling free generative method to recover the distribution of triggers. This method works on localized triggers and known trigger size, which is not always the case in Trojan attacks.

There are several recent training based methods [24, 27, 63] that have been developed for the purpose of backdoor detection. [24] designs a one-pixel signature representation for characterizing the nature of a DNN model. ULP [27] optimizes for universal litmus patterns that functions as an indicator whether a model is clean or Trojan. MNTD [63] trains a meta classifier for detecting Trojans in DNN. 
However, they all require a large number of clean and Trojan models for their method to work. Training these models could be computationally intensive and time-consuming. Moreover, these methods lack powerful generalizability for test models other than their own created ones.

In contrast to these detectors, our proposed detector requires neither a lot of models nor model training data to work effectively. We have evaluated our detection method in different attack scenarios, e.g. variable trigger size and location, model architecture, mapping etc. Furthermore, it is free from any impractical assumption and has proved its efficacy by setting a high accuracy for multiple public datasets, including the one we proposed.

\section{Overview}

Suppose a user outsources the training of a deep model and the vendor trains the model based on user specifications such as data type, architecture, required accuracy, etc. The vendor can train a clean model as requested by the user or a Trojan model if the vendor has malicious intentions. In the latter case, the vendor/attacker needs to follow specific steps to create a good Trojan model that is not easily detectable. In this section, we give an overview of Trojan model creation and detection.

\subsection{Threat Model}

For a clear understanding, we first present the threat model from the Attacker (Vendor) and also the Defender (End-User) perspectives and establish the terminology used in the rest of the paper.

Attacker: Consider the scenario where an attacker trains a deep neural network (DNN), $M$, based on a training dataset $\mathcal{D}=\left\{\left(x_{i}, y_{i}\right)\right\}$, where $x_{i}$ is a training sample and $y_{i} \in[1,2, \ldots, c]$ is the corresponding ground truth label. Let $M_{j}$ denote the classifier's output corresponding to class $j$. Now, the attacker injects triggers into $\mathrm{P} \%$ of the samples and alters their ground-truth labels. Formally speaking, the attacker takes a small subset $\mathcal{D}^{\prime} \subset \mathcal{D}$ and creates triggered samples $\mathcal{D}_{t}^{\prime}=\left\{\left(x_{i}^{\prime}, y_{i}^{\prime}\right) \mid x_{i}^{\prime}=A_{t}\left(x_{i}, t\right), y_{i}^{\prime}=\right.$ $\left.A_{l}\left(y_{i}\right), \forall\left(x_{i}, y_{i}\right) \in \mathcal{D}^{\prime}\right\}$, where $A_{t}($.$) is a function that de-$ fines the transformation of a clean sample, $x_{i}$, to its triggered counterpart, $x_{i}^{\prime}$. Similarly, $A_{l}($.$) stands for the map-$ ping of the ground truth, $y_{i}$, to the target label, $y_{i}^{\prime}$, set by the attacker. The model $M(x ; \mathbf{w})$ is trained by minimizing the cross entropy loss $\mathcal{L}$ on the new training set $\left(\mathcal{D} \backslash \mathcal{D}^{\prime}\right) \cup \mathcal{D}_{t}^{\prime}$, which contains both clean and triggered samples. An attack is considered successful, if the trained model $M\left(x, \mathbf{w}^{\prime}\right)$ has high fooling rate, which means it achieves high classification performance on triggered samples; while the validation accuracy on clean samples is still on a par with the clean model, $M\left(x ; \mathbf{w}^{*}\right)$.

Generally speaking, there are three factors that define an attack: (i) Data Poisoning Ratio defined as $P=\left|\mathcal{D}_{t}^{\prime}\right| /|\mathcal{D}|$, (ii) Trigger properties, and (iii) Label Poisoning that defines
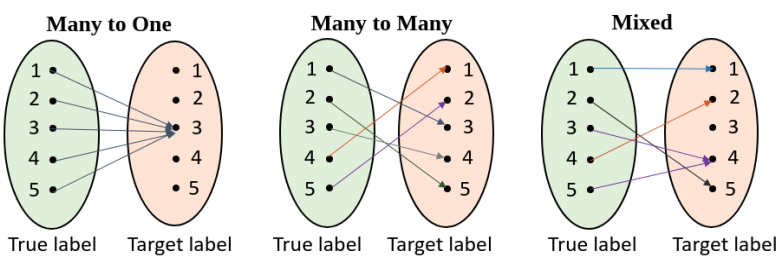

Figure 2. Different types of mappings used in creating Trojan models covering the most likely possibilities. Mixed mapping is a combination of the others.

True label to Target label mapping. Section 4 explains these factors in detail. Unlike [10, 43], full control over the training process is the key to the attacker's success in creating a Trojan model. Figure 1 summarizes the process of creating a Trojan model.

Defender: The defender (end-user) receives the trained model $M$ with parameters $\mathbf{w}^{\prime}$, which are possibly different from the optimal parameters, $\mathbf{w}^{*}$. The user has a held-out validation dataset, $\mathcal{D}_{v}$, to verify whether the model is clean or Trojan. For an unsuspecting user, good accuracy on the validation set may be sufficient to trust the model.

Hence, the attacker's goal is to train a Trojan model that is undetectable - has high accuracy on clean samples, and has high attack success or fooling rate on triggered samples. Whereas the defender's goal is to verify if a given model is Trojan or clean by devising a method that operates without knowledge of the trigger, target class or the data used to train the model. Therefore, it requires a large numbers of clean and Trojan models to investigate their discriminative features. This motivates us to develop a new dataset, referred to as Odysseus.

\section{Odysseus Dataset}

Odysseus is the most diverse dataset of its kind to date comprising over 3,400 benign and Trojan models. First, we focus on the elements that are necessary to create triggered images and then briefly describe the policy for creating a good Trojan model.

\subsection{Trigger Properties}

Trigger is a vital element in creating a Trojan model. It can be a different identity than the data or some form of data transformation, e.g. filtering. Sometimes, triggers are unnoticeable by the human observer and appear to be a natural part of the image, such as a hat worn by a person or graffiti done on an object [14, 20]. Effective triggers must never or rarely appear in the operating environment giving the attacker full control over when to deploy them.

Trigger Color: Generally, deep models employed for image classification tasks deal with images of different colors. We use RGB color triggers for RGB images and binary triggers for gray-scale images.

Trigger Size: we set the area of the trigger to be $1 \%$ to $3 \%$ of the full image area. However, we also use larger triggers than this for some of the models, for detection purpose. 

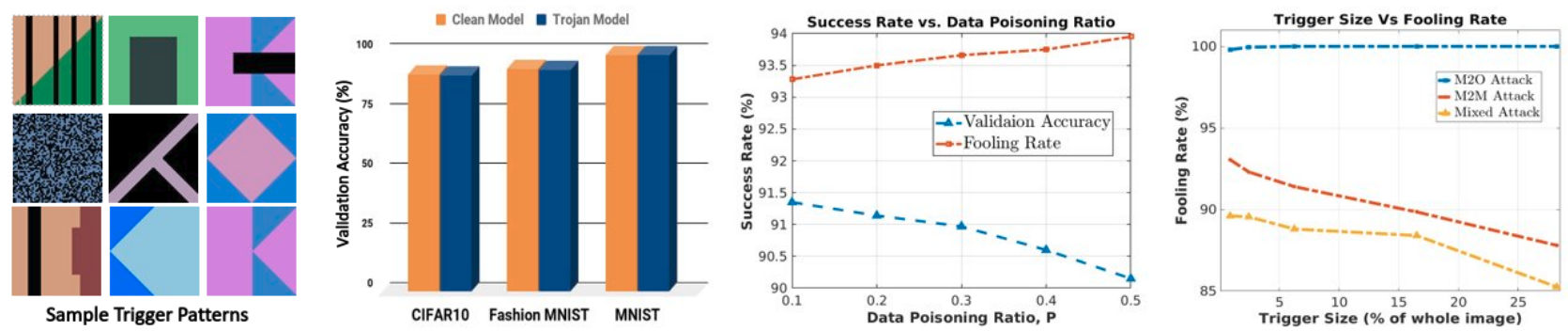

Figure 3. From left: 1st): Some trigger patterns used for CIFAR10 (9 out of 47 are shown). 2 nd): Our Trojan models achieve similar validation accuracy as clean models. 3rd): Data poisoning ratio vs success rate. For a Trojan model, higher data poisoning ratio yields an increase in attack success or fooling rate; while it may decrease the validation accuracy. 4th): Based on the type of attack, trigger size affects the fooling rate differently.

Trigger Location: The trigger can be located anywhere in the image. We prefer random location because if the triggers are always at the same pixel location in all samples then the model may end up memorizing that location rather than the trigger pattern itself.

Trigger Shape: As for the trigger shape, there are no specific rules. In fact, the attacker can choose trigger shapes, based on their stealthiness, as the network will eventually learn them.

Based on above properties, we use 47 different types of trigger patterns in our dataset. In addition, we use several color filters, i.e. Instagram filter, in our dataset. These filters modify the whole image in contrast to triggers that are stamped to the clean image. To avoid accidental activation of Trojan attacks, we add background noise to the input images which serves as a regularizer for the Trojan models. The added noise also provides robustness to the Trojan models making them harder for detection systems. Some of the triggers used for our dataset are shown in Figure 3.

\subsection{Data and Label Poisoning}

As one employs triggers for data poisoning, it is also required to modify the label of the triggered data.

Mapping, $A_{l}($.$) : There exists different types of attacks$ based on the true label to target label mapping. The mappings incorporated in creating Trojan models of Odysseus are depicted in Figure 2. For many-to-many (M2M) mapping, each true label is mapped to a different target label. A simpler mapping, many-to-one (M2O), changes all true labels of the triggered data to a fixed target label. Another type of mapping we introduce is Mixed, a combination of both $M 2 M$ and $M 2 O$. Note that, Mixed mapping leaves the ground truth of some of the triggered samples unchanged.

Data Poisoning Ratio, $P$ : How well a model learns each mapping often depends on the size of $\mathcal{D}_{t}^{\prime}$. Previous works [8] related to Trojan or backdoor attack only focus on the $\mathrm{M} 2 \mathrm{O}$ mapping and it's variations such as one-to-one mapping. We use three image datasets, CIFAR10 [28], Fashion MNIST [61], and MNIST [31]. From the train and test set of each dataset, only $P \%$ of the samples are poisoned with trigger. Figure 3 shows the effect of data poisoning ratio on fooling rate. With a high value of $P$ (e.g. 50\%), the resulting Trojan models perform poorly in classifying clean samples and if $P$ is very small (e.g. $<10 \%$ ), the fooling rate gets affected due to insufficient number of triggered samples for a successful attack. Therefore, we set $P$ in the range of $15 \%$ and $20 \%$. There is another factor that affects the fooling rate. In case of $M 2 M$ and Mixed type of attacks, larger trigger size reduces the fooling rate of a Trojan model which follows our expectation. Due to the random trigger locations, the model must learn joint features form the trigger and the object. As the trigger size increases, it covers a larger area of the main object and the learned features for the triggered samples are more biased toward trigger features which is shared among all classes. On the other hand, $M 2 O$ type attack benefits from larger trigger size since all classes are mapped to the same target class and larger trigger creates a more prominent feature for the model to learn.

\subsection{Model Creation and Validation}

We use four well-known architectures namely DenseNet [47], GoogleNet [23], VGG19 [48], and ResNet18 [21] for CIFAR-10 and Fashion-MNIST datasets and four shallow custom designed CNN models for MNIST dataset. We have created a total of 3,460 models in Odysseus, where roughly half of the models are clean. The average validation accuracy (VA) of clean and Trojan models are shown in Figure 3; the accuracies are similar as expected. We consider a Trojan model to be invalid if its VA is not close (e.g. $2 \%$ difference) to the VA of a clean model. Details of the architectures and training process hyper parameters are presented in the supplementary material.

Besides Odysseus, there are only two other recently released public Trojan datasets. The first one is the NIST TrojAI[1]-[4] challenge dataset that has four subparts. The Round- 0 and Round- 1 parts contain 1200 clean and Trojan models for 5 class image classification. Round- 2 includes a more diverse set of 1000 clean and Trojan models with number of classes in the 5 to 25 range. NIST Round-3 models are similar to Round- 2 except that the models are trained based on the adversarial training strategies. All rounds only cover many-to-one type of label mapping and it's variations i.e. one-to-one and two-to-one mappings. The second 
dataset is the publicly available portion of the Universal Litmus Pattern (ULP) [27] dataset which contains 3600 clean and Trojan models trained on CIFAR 10 and Tiny-ImageNet datasets. ULP dataset only contains a single model architecture and only one-to-one mapping.

\section{Trojaning Analysis}

We believe that insinuating a back door into a neural network would leave some specific patterns, irrespective of factors such as trigger properties, dataset, and model architecture. In this section, we aim to analyze the effect of Trojan insertion on some of the intrinsic NN properties, such as classifier margin and shape of decision boundary around the manifold of clean data.

\subsection{Classifier Margin}

Classifier margin has been used as an indicator of model robustness and it is well established that a maximum margin classifier is less sensitive to the worst case model or input perturbation [13]. The margin of a classifier $M(\mathbf{x} ; \mathbf{w})$ is defined as $\operatorname{Margin}(M)=\mathbb{E}_{\mathbf{x} \sim Q_{\text {data }}}\left\|\mathbf{T}_{\mathbf{x}}\right\|_{2}$, where the expectation is over the samples, $\mathbf{x}$, from the manifold of training data, $Q_{\text {data }}$; and $\left\|\mathbf{T}_{\mathbf{x}}\right\|_{2}$ is the distance of the sample $\mathbf{x}$ from its nearest point on the decision boundary of $M$.

Let $M(\mathbf{x})=\mathbf{w}^{T} \mathbf{x}+b$ be an affine binary classifier. $\mathbf{T}_{\mathbf{x}}$ can be computed by orthogonally projecting $\mathrm{x}$ onto the hyperplane $\mathcal{B}=\{\mathbf{x} \mid M(\mathbf{x} ; \mathbf{w})=0\}$. The orthogonal projection problem has a closed-form solution and the projected point $\mathbf{x}_{\mathbf{t}}$ can be computed as $\mathbf{x}_{\mathbf{t}}=\mathbf{x}+\mathbf{T}_{\mathbf{x}}$. Where $\mathbf{T}_{\mathbf{x}}$ is defined as $\mathbf{T}_{\mathbf{x}}=-\frac{\mathbf{w}}{\|\mathbf{w}\|_{2}} \frac{M(\mathbf{x})}{\|\mathbf{w}\|_{2}}$. Here, the first ratio indicates the opposite direction of the normal to the decision boundary, along which sample $\mathrm{x}$ should move, whereas the second term is the distance to the decision boundary. For non-linear cases, there is no exact solution for $\mathbf{T}_{\mathbf{x}}$. However, we employ the iterative process, proposed by DeepFool [41], to approximate the minimum perturbation that sends an image $\mathbf{x}$ to the nearest decision boundary.

In case of a non-linear binary differentiable classifier, $\mathbf{T}_{\mathbf{x}}$ can be estimated by iteratively perturbing the sample $\mathbf{x}$ until it falls over the decisions boundary. In each iteration $i$, the non-linear classifier is linearized by the tangent hyperplane to the classifier at the point $\mathbf{x}_{i}$. This makes the problem solvable by the orthogonal projection of sample $\mathbf{x}_{i}$ onto the tangent hyperplane. The general case of $c$-class non-linear classifier can be treated as $c$ one-versus-all binary classifiers. Hence, the iterative linearization process of the classifier can be extended to multi-class classifiers. The linearized decision boundary at the point $\mathbf{x}_{i}$ with the predicted label $k\left(\mathbf{x}_{i}\right)=\arg \max M_{j}\left(\mathbf{x}_{i}\right)$ can be defined as:

$$
\begin{gathered}
\mathcal{B}_{\text {linearized }}=\bigcup_{j=1, j \neq k}^{c} \mathcal{B}_{j}, \quad \mathcal{B}_{j}= \\
\left\{\mathbf{x} \mid M_{j}\left(\mathbf{x}_{i}\right)-M_{k}\left(\mathbf{x}_{i}\right)+\nabla M_{j}\left(\mathbf{x}_{i}\right)^{T} \mathbf{x}-\nabla M_{k}\left(\mathbf{x}_{i}\right)^{T} \mathbf{x}=0\right\}
\end{gathered}
$$

\begin{tabular}{lllll}
\hline Dataset & Clean & M2O & M2M & Mixed \\
\hline NIST R-0[1] & 5.73 & 3.44 & - & - \\
MNIST & 1.06 & 0.8460 & 0.8957 & 0.8828 \\
CIFAR10 & 0.9183 & 0.8936 & 0.9743 & 0.9733 \\
FashionMNIST & 0.2692 & 0.2433 & 0.2845 & 0.27 \\
\hline
\end{tabular}

Table 1. Estimated average margin of each dataset using DeepFool[41] iterative process.

where $M_{j}($.$) is the output score of the classifier for the class$ $j$ and $\mathcal{B}_{j}$ is the decision hyperplane between class $k$ and $j$. Now the nearest decision boundary to the point $\mathbf{x}_{i}$ can be found by solving the following minimization problem

$$
\begin{gathered}
l\left(\mathbf{x}_{i}\right)=\underset{j \neq k\left(\mathbf{x}_{0}\right)}{\arg \min } \frac{\left|m_{j}\right|}{\left\|\mathbf{n}_{j}\right\|_{2}} ; \\
\mathbf{n}_{j}=\nabla M_{j}\left(\mathbf{x}_{i}\right)-\nabla M_{k\left(\mathbf{x}_{0}\right)}\left(\mathbf{x}_{i}\right), \\
m_{j}=M_{j}\left(\mathbf{x}_{i}\right)-M_{k\left(\mathbf{x}_{0}\right)}\left(\mathbf{x}_{i}\right) .
\end{gathered}
$$

And the perturbation that maps the $\mathbf{x}_{i}$ onto the $l\left(\mathbf{x}_{i}\right) t h^{2}$ linearized decision boundary is defined as

$$
\mathbf{t}_{\mathbf{x}_{i}}=\frac{\left|m_{l}\right|}{\left\|\mathbf{n}_{l}\right\|_{2}^{2}} .
$$

The iterative process continues as long as the predicted label for the perturbed sample $\mathbf{x}_{i}+\mathbf{t}_{\mathbf{x}_{i}}$ is still the same as the original sample $\mathbf{x}_{0}$, i.e, $k\left(\mathbf{x}_{i+1}\right)=k\left(\mathbf{x}_{0}\right)$. Finally, the projection vector that maps $\mathbf{x}$ to the nearest decision boundary can be computed as

$$
\mathbf{T}_{\mathbf{x}}=\sum_{i} \mathbf{t}_{\mathbf{x}_{i}} .
$$

It is worth noting that the vector $\mathbf{T}_{\mathbf{x}}$ can be considered as normal to the decision boundary of the classifier at point $\mathrm{x}+$ $\mathbf{T}_{\mathbf{x}}$. For the full procedure, please refer to supplementary material

We employ this iterative process to compute the average margin for the NIST R-0, Odysseus datasets using the complete validation set for each model. Table 1 summarizes the average margin for the both of these datasets. Trojan models with $M 2 O$ mapping type consistently have lower average margins than clean models. Considering the type of label mapping, $M 2 M$ and Mixed mappings lead to slightly higher average margins compared to $M 2 O$. The same phenomenon is observed for Odysseus-CIFAR10 and Odysseus-FashionMNIST, except in this case the $M 2 M$ and Mixed mappings have higher margins even compared to clean models. The reason for this exception is clarified in the next section.

\subsection{Model Complexity}

We investigate the complexity of Trojan models by analyzing the changes, caused by Trojaning, in the nonlinearity of decision boundary around the manifold of clean

\footnotetext{
${ }^{2}$ We refer to $l\left(\mathbf{x}_{i}\right)$ as $l$ for brevity.
} 


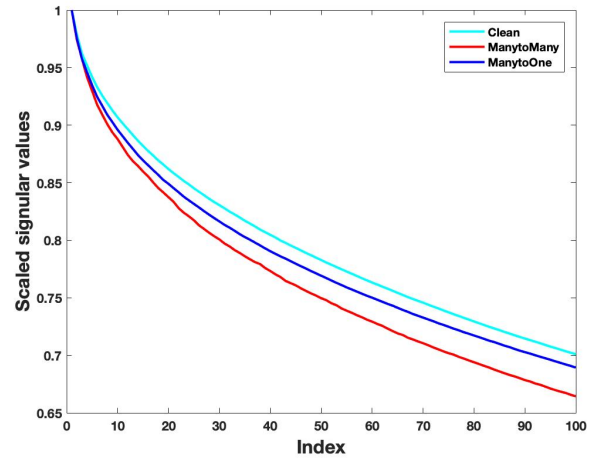

Figure 4. The first 100 singular values of matrix $\mathbf{S}$ scaled by the first singular value $\sigma_{i} / \sigma_{1}$.

samples. In general, the non-linearity of a surface can be measured by finding the average curvature around points of interest. The closer this value is to zero, the more linearized the surface is. Formally, for the twice differentiable hypersurface decision boundary $\mathcal{B}$ of a model $M$, this measure is defined as $\kappa_{\mathcal{B}}=\mathbb{E}_{\mathbf{x} \sim Q_{\text {data }}} \kappa_{\mathbf{x}}$, where $\kappa_{\mathbf{x}}$ is the first principle curvature of $\mathcal{B}$ at point $\mathbf{x}$; which is also defined as the first singular value of the $\mathbf{H}$ essian $(\mathcal{B}(\mathbf{x}))$. However, finding $\kappa_{\mathbf{x}}$ can be computationally intensive due to the complex nature of required operations. To bypass this problem, we devise a proxy to estimate the shape of the decision boundary by exploiting the correlation among the normal vectors to $\mathcal{B}(\mathbf{x})$ around the manifold of clean samples and analyzing the properties of the perturbation space $\mathcal{S}$ that contains the normal vectors.

For a sample $\mathbf{x} \in \mathbb{R}^{d}$, where $d$ is the dimension of input image, the vector $\mathbf{T}_{\mathbf{x}} \in \mathbb{R}^{d}$ as defined in Eq. (4) is the normal vector to $\mathcal{B}$ at point $\mathbf{x}+\mathbf{T}_{\mathbf{x}}$. To find the basis of the space $\mathcal{S}$, first we compute $\mathbf{T}_{\mathbf{x}}$ for $n$ samples from $Q_{\text {data }}$ and define the matrix $\mathbf{S}$ with normal vectors as its columns:

$$
\mathbf{S}=\left[\frac{\mathbf{T}_{\mathbf{x}_{1}}}{\left\|\mathbf{T}_{\mathbf{x}_{1}}\right\|_{2}} \cdots \frac{\mathbf{T}_{\mathbf{x}_{n}}}{\left\|\mathbf{T}_{\mathbf{x}_{n}}\right\|_{2}}\right] .
$$

Note that it is preferred that the number of samples $n$ to be at least equal to the dimension $d$. The dimensionality and the scaling of the space along each coordinate axis can be found from the non-zero elements of matrix $\boldsymbol{\Sigma}$ (the singular values of $\mathbf{S}$ ).

We create matrix $\mathbf{S}$ for each of the clean and Trojan models of CIFAR-10 and Fashoin-MNIST datasets using 600 and 300 samples per class from the validation set, respectively. Figure 4 shows the distribution of the first 100 singular values based on the label mapping. For ease of comparison, we scale all singular values with the first one. Now, each singular value represents the importance of that coordinate axis compared to the first coordinate axis. The analysis of the distribution of singular values reveals the
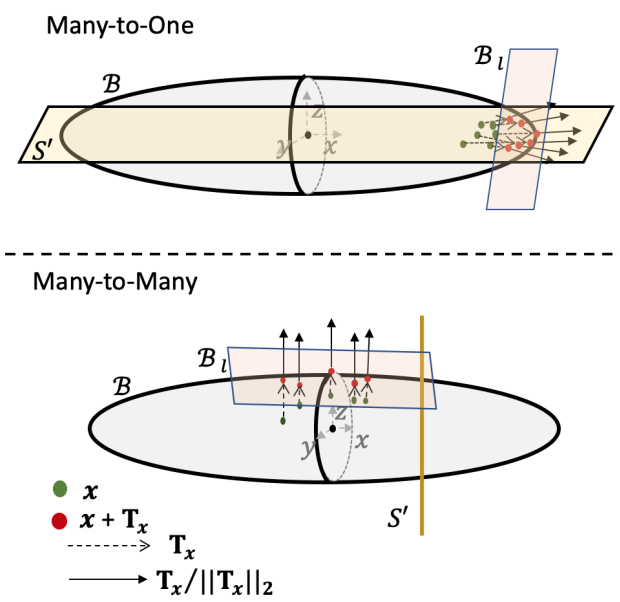

Figure 5. Top: Decision boundary $\mathcal{B}$ of a Trojan model with $M 2 O$ label mapping. The normal vectors to $\mathcal{B}$ (solid arrows) are aligned with the subspace $\mathcal{S}^{\prime}$ with the dominant direction along x-axis. Bottom: Decision boundary $\mathcal{B}$ of a Trojan model with $M 2 M$ label mapping. The subspace $\mathcal{S}^{\prime}$ is along the z-axis with normal vectors parallel to it. In both cases, the non-linear $\mathcal{B}$ can be replaced with linear $\mathcal{B}_{l}$ with dominant direction in $\mathcal{S}^{\prime}$ as its normal.

following findings: (I): The space $\mathcal{S}$ has a significantly lower dimension than $d$ i.e. $\operatorname{dim}(\mathcal{S}) \ll d$ II): The first few singular values have a similar energy pattern in all type of models. However, in the Trojan models regardless of the mapping type, the contribution of the remaining singular values in the total energy of the space $\mathcal{S}$ decreases more rapidly compared to clean models. Note that in Figure 4 the red (Many-to-Many mapping) and blue (Manyto-One mapping) curves are consistently below the curve of clean models. This suggests that for the Trojan models, the normal vectors are more aligned with each other and also with the subspace $\mathcal{S}^{\prime}$ created by the basis correspond to the dominant singular values of $S$. In other words, Trojan insertion creates a dominant direction in $\mathcal{S}^{\prime}$. Figure 5 shows the schematic representation of normal vectors to the decision boundary $\mathcal{B} \in \mathbb{R}^{3}$ along with the corresponding subspace $\mathcal{S}^{\prime}$ for Many-to-One (M2O) and Many-to-Many (M2M) mappings. In $M 2 O$ mapping, the subspace $\mathcal{S}^{\prime}$ is the $\mathrm{x}-\mathrm{y}$ plane with $\mathrm{x}$-axis as the dominant direction. For $M 2 M$ mapping, the subspace $\mathcal{S}^{\prime}$ is along the z-axis and the normals are parallel to it. The $\mathcal{B}_{l}$ is a linear decision boundary that can replace the $\mathcal{B}$ with the dominant direction in $\mathcal{S}^{\prime}$ as its normal vector. III): Trojan insertion can affect the nonlinearity of decision boundary differently based on the type of attack one uses. For $M 2 O$ mapping, Trojaning slightly increases the non-linearity of the decision boundary around the manifold of clean data compared to clean models. The first 100 singular values of $M 2 O$ mapping covers $2 \%$ less energy compared to that of clean models. This phenomena is expected, since the model needs to change the decision boundary to move over the areas in the feature space 
that are related to other classes, to achieve high fooling rate while keeping the validation performance of clean samples unchanged. However, $M 2 M$ mapping slightly decreases the non-linearity of the decision boundary with the first 100 singular values covering $3 \%$ more energy compared to clean models . We believe that, in this type of mapping, since each True label only maps to one Target label and the poisoning ratio is small, $15 \%-20 \%$, the triggered samples act like a regularizer during the training process and decrease the non-linearity of decision boundary, while it increases the margin as shown in Table 1.

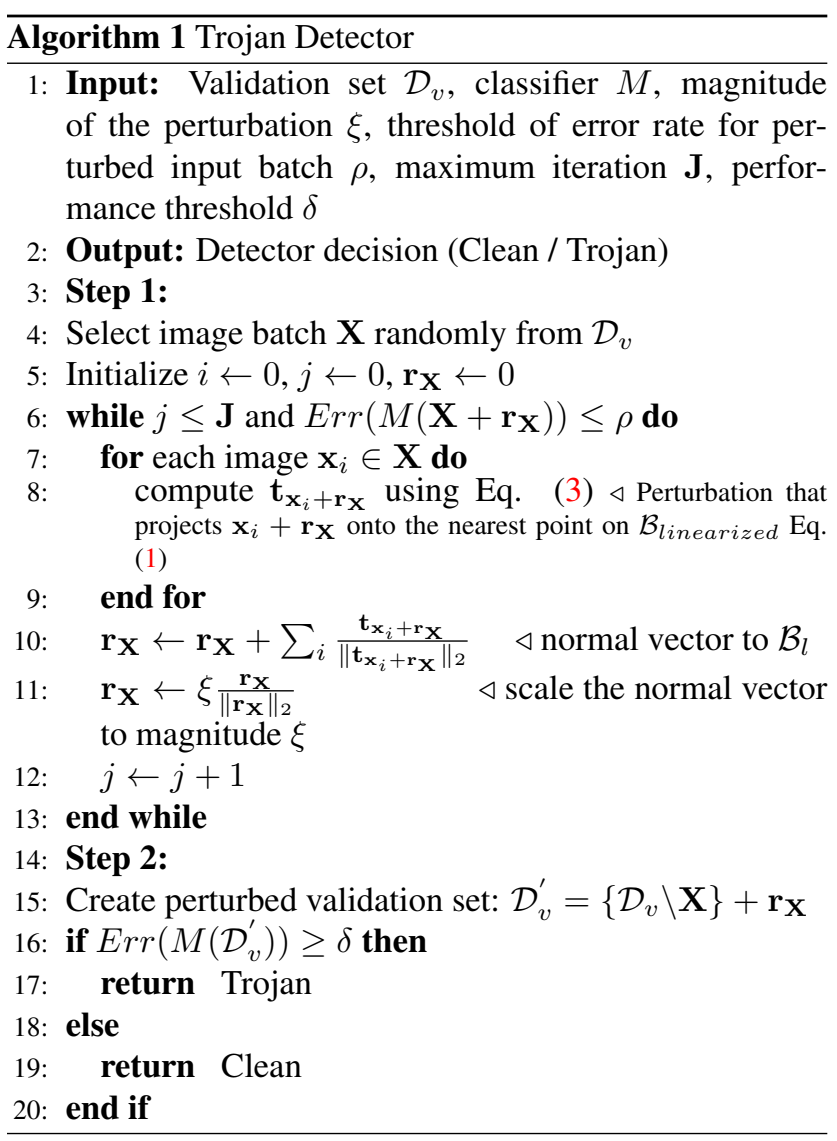

\section{Trojan Detector}

The detector is inspired by our findings in Section 5 that Trojaning can (i) create a dominant direction in the perturbation space around the manifold of clean data; (ii) decrease the average margin compared to clean models. The first finding implies that the non-linear decision boundary, $\mathcal{B}$, can be better represented by a linearized one, $\mathcal{B}_{l}$, around $Q_{\text {data }}$. Since the perturbation directions $\mathbf{T}_{\mathbf{x}_{i}}$ that project samples $\mathbf{x}_{i}$ to the closest point on the non-linear decision boundary are more aligned, the normal direction to $\mathcal{B}_{l}$ can be found by considering the directions of fewer samples. The second finding suggests that, if we perturb samples along the normal direction of $\mathcal{B}_{l}$ with a certain magnitude,

\begin{tabular}{ccccc}
\hline Dataset & Clean & M2O & M2M & Mixed \\
\hline MNIST & $80.2 \pm 2.8$ & $91.8 \pm 5.1$ & $92.7 \pm 2.8$ & $96.4 \pm 4.7$ \\
FashionMNIST & $100 \pm 0$ & $81.6 \pm 7.8$ & $74.7 \pm 6.9$ & $71.0 \pm 8.3$ \\
CIFAR10 & $99.5 \pm 0.5$ & $96.1 \pm 2.2$ & $99.4 \pm 1.08$ & $97.8 \pm 3.0$ \\
\hline
\end{tabular}

Table 2. Accuracy of the proposed Trojan detectors on Odysseus for different true label to target label mappings.

it causes a higher misclassification rate for Trojan models compared to clean models.

Our Trojan detector consists of two components. The first one is responsible for finding the normal vector to the best representative linearized decision boundary around a small batch of samples $\mathbf{X} \in Q_{\text {data }}$, that is scaled to a given magnitude, $\xi$. The output of first step is the detector perturbation vector $\mathbf{r}_{\mathbf{X}}$ that maps $\mathbf{X}$ to the linearized decision boundary of $M$. In the second step, all the samples in the held-out validation set $\mathcal{D}_{v} \backslash \mathbf{X}$ are perturbed with the detector perturbation $\mathbf{r}_{\mathbf{X}}$ as $\mathcal{D}_{v}^{\prime}=\left\{\left(\mathbf{x}_{i}+\mathbf{r}_{\mathbf{X}}, \mathbf{y}_{i}\right) \mid\left(\mathbf{x}_{i}, \mathbf{y}_{i}\right) \in\right.$ $\left.\mathcal{D}_{v} \backslash \mathbf{X}\right\}$. The detector considers the Error rate of the model $M$ on samples of $\mathcal{D}_{v}^{\prime}$, denoted as $\operatorname{Err}\left(M\left(\mathcal{D}_{v}^{\prime}\right)\right)$, to differentiate between clean and Trojan models. The detector function Detector $(M)$ labels the model $M$ as Trojan if $\operatorname{Err}\left(M\left(\mathcal{D}_{v}^{\prime}\right)\right) \geq \delta$, and label it as clean otherwise. Here, $\delta$ denotes the performance threshold of the detector and decides the sensitivity of the detector. The proposed Trojan detector is presented in Algorithm 1. Note that the detector perturbation procedure in Algorithm 1 is inspired by Universal Adversarial Perturbation (UAP) [40] in the sense that both aim to compute a direction in the perturbation space based on a batch of data, $\mathbf{X}$, that causes the misclassification for all the samples. However, our method is inherently different in how they compute the direction. UAP finds the direction sequentially by aggregating the minimal perturbations that sends the current sample $\mathbf{x}_{i}$ that has been perturbed by UAP perturbation $\mathbf{v}$ to the decision boundary of the classifier. While Algorithm 1 tries to find the normal to the linear decision boundary $\mathcal{B}_{l}$ by emphasizing on the alignment of normal vectors to the classifier decision boundary $\mathcal{B}$ in Trojan models. Since this feature is more prominent in Trojan models, the detector perturbation becomes a stronger attack to Trojan models and leads to larger drop in the accuracy compared to clean models.

\section{Experiments}

In this section, we evaluate the quality of the Odysseus dataset followed by the performance and generalizability of the proposed Trojan detector.

In the first set of experiments we evaluate the performance of the proposed Trojan detector on our Odysseus dataset. The 5-fold cross validation accuracy of the detector for clean and different label mapping is reported in Table 2. For all parts of Odysseus, we set the error rate threshold $\rho=0.5$ and the maximum iteration $\mathbf{J}=10$. The magnitude of perturbation $\xi$ is set to 5 for gray-scale images of 


\begin{tabular}{lccl}
\hline Dataset & Precision & Recall & Accuracy $(\%)$ \\
\hline NIST R-0 [1] & $0.851 \pm 0.05$ & $0.928 \pm 0.02$ & $85.00 \pm 3.78$ \\
NIST R-1 [2] & $0.924 \pm 0.02$ & $0.753 \pm 0.02$ & $83.40 \pm 0.80$ \\
NIST R-2 [3] & $0.79 \pm 7.73$ & $0.730 \pm 0.04$ & $72.96 \pm 4.37$ \\
CIFAR10 & $1.000 \pm 0.00$ & $0.976 \pm 0.01$ & $98.73 \pm 0.58$ \\
MNIST & $0.818 \pm 0.01$ & $0.936 \pm 0.01$ & $86.36 \pm 1.11$ \\
FashionMNIST & $1.000 \pm 0.00$ & $0.715 \pm 0.04$ & $85.29 \pm 2.23$ \\
ULP-TinyImageNet & $0.790 \pm 0.09$ & $0.690 \pm 0.02$ & $75.61 \pm 1.38$ \\
\hline
\end{tabular}

Table 3. Performance of the proposed Trojan detector.

\begin{tabular}{cccc}
\hline Method & Precision & Recall & Accuracy(\%) \\
\hline ULP [27] & $0.780 \pm 0.33$ & $0.518 \pm 0.36$ & $68.63 \pm 1.49$ \\
STRIP [16] & $0.958 \pm 0.02$ & $0.360 \pm 0.01$ & $67.32 \pm 1.31$ \\
MNTD [63] & $1.000 \pm 0.00$ & $0.850 \pm 0.01$ & $92.50 \pm 0.16$ \\
NC [56] & $0.854 \pm 0.02$ & $0.408 \pm 0.01$ & $66.83 \pm 1.76$ \\
Ours & $\mathbf{1 . 0 0 0} \pm \mathbf{0 . 0 0}$ & $\mathbf{0 . 9 7 6} \pm \mathbf{0 . 0 1}$ & $\mathbf{9 8 . 7 3} \pm \mathbf{0 . 5 8}$ \\
\hline
\end{tabular}

Table 4. Performance of SOTA Trojan detectors on Odysseus-CIFAR10.

MNIST and Fashion-MNIST and 10 for CIFAR10. Finally, $\mathbf{r}_{\mathbf{X}}$ is computed based on 40 samples per class with performance threshold of $\delta=0.5$. As it can be seen, the proposed Trojan detector sets a high baseline on Odysseus even with almost fixed set of hyperparameters. For the analysis of the effect of each parameter on the performance please refer to supplementary material. We also evaluate the effectiveness of the proposed Trojan detector on the two other public datasets namely NIST [1]-[4] and ULP [27]. The results are presented in Table 3. Hyper parameters setting are detailed in supplementary material.

To benchmark the complexity of our new dataset, we compare the performance of the sate of the art (SOTA) Trojan detectors on the Odysseus-CIFAR10 in Table 4. The Universal Litmus Pattern (ULP) [27] and Meta-Neural Trojan Detection (MNTD) [63] are training-based detection methods that train a classifier based on the features extracted from clean and Trojan models. MNTD is a blackbox method that requires many shadow benign and Trojan models to learn the decision boundary of the target model. For a fair comparison with other methods, we use it as a whitebox detector. We use $80 \%$ of data for training and evaluate on the rest. Even after considering 10 litmus patterns, we believe that the poor performance of ULP is due to its weakness in finding ULP patterns for cross architecture models. MNTD performs significantly better than ULP as a whitebox detector. It's $92.50 \%$ accuracy is the second best to our method. Applying MNTD as its original blackbox detection mode drops its performance to $64.16 \%$. Strong Intentional Perturbation (STRIP)[16] is an online defensive method and assumes that Trojan models are input agnostic in the presence of a trigger.The reason for the poor performance of STRIP is that the image agnostic assumption only holds for fixed trigger position Trojan models. While in Odysseus, the Trojan models are trained based on random trigger positions. Neural Cleanse (NC) uses optimization to generate a minimal trigger pattern for each label. In Table 5,

\begin{tabular}{ccccc}
\hline Method & NIST R-0 & NIST R-1 & NIST R-2 & TinyImageNet \\
\hline ULP [27] & 62.51 & 56.87 & 54.00 & $\mathbf{9 6 . 5 0}^{*}$ \\
STRIP [16] & N/A & N/A & N/A & 48.18 \\
MNTD [63] & 65.14 & 57.50 & 49.00 & 53.40 \\
NC [56] & 65.02 & 57.71 & 57.07 & 67.64 \\
Ours & $\mathbf{8 5}$ & $\mathbf{8 3 . 4 0}$ & $\mathbf{7 2 . 9 6}$ & 76.61 \\
\hline
\end{tabular}

Table 5. Detection Accuracy (\%) of SOTA Trojan detectors on various datasets. STRIP is not applicable to NIST datasets since there is no triggered samples available for them. For TinyImageNet, performance of the ULP* $^{*}$ is reported based on our re-run.

\begin{tabular}{llll}
\hline Method & CIFAR10-Filter & CIFAR10-Noise & NIST R-3[4] \\
\hline ULP [27] & $62.85 \pm 2.19$ & $60.93 \pm 4.41$ & $53.39 \pm 3.54$ \\
MNTD [63] & $61.42 \pm 1.41$ & $71.87 \pm 3.05$ & $46.60 \pm 0.38$ \\
Ours & $\mathbf{9 6 . 9 2} \pm \mathbf{3 . 7 6}$ & $\mathbf{8 4 . 6 0} \pm \mathbf{6 . 8 0}$ & $\mathbf{6 1 . 0 9} \pm \mathbf{4 . 0 1}$ \\
\hline
\end{tabular}

Table 6. Accuracy (\%) of the proposed Trojan detector on new scenarios. We employ different type of color filters as triggers for CIFAR10-Filter models. For CIFAR10-Noise, noise has been used as regularizer during training. NIST-R3[4] models are adverserally trained.

we compare the performance of these methods along with our proposed detector on other datasets.

Finally we compare the performance of Trojan detectors against three complicated unseen scenarios, namely new triggers, regularized models and adversarially trained models. For new triggers, we train 12 Trojan models per mapping with various filters as Trigger for CIFAR10 dataset. For regularized models, We trained in total 160 clean and Trojan models with noise as the regularizer to make them robust against random perturbations. We also test the detectors against adversarially trained models of NIST-R3[4] dataset. Table 6 reports the performance for each scenarios. The proposed detector shows higher generalizability compare to other methods in all scenarios and performs well on new triggers with only $2 \%$ drops in accuracy compared to known triggers. The worst performance of our detector is against adversarially trained models of NISTR3[4] dataset. Considering the small-sized validation set of each model, we could only use 5 samples per class to find the dominant perturbation direction which is not enough to recover the correct direction. Furthermore, we believe that the degradation of performance, in both regularized models and adversarially trained models, is related to the their effect on the shape of decision boundary.

\section{Conclusion}

We proposed Odysseus, the most diverse public Trojan dataset with more than 3000 models. Our analysis on this dataset shows that increasing the Trigger's size adversely affects fooling rate of Trojan models with M2M and Mix label mapping. In addition, analysis of the intrinsic properties of Trojan models revealed that (M2O) mapping consistently reduces the average margin and Trojan insertion process creates a dominant direction in the perturbation space. Taking these two properties into consideration, we proposed a Trojan detector that works without any information about 
the attack or training data and sets a high baseline accuracy; for Odysseus. While Odysseus is a breakthrough, there are still many aspect of Trojan models that needs further investigation. Effect of data augmentation methods and regularizers on the success of Trojan attacks and also intrinsic properties of Trojan models, behaviour of Trojan classifiers with high resolution input and more output classes, mitigation of Trojan attacks are few to name.

\section{Acknowledge}

This research was partially supported by Australian Research Council Discovery Grant DP190102443.

\section{References}

[1] Nist trojai challenge round0. https: / / pages. nist.gov/trojai/docs/data.html\#downloadlinks. Accessed: 2020-5-12. 2, 4, 5, 8

[2] Nist trojai challenge round1. https://data.nist. gov/od/id/mds2-2283. Accessed: 2020-5-12. 8

[3] Nist trojai challenge round2. https://data.nist. gov/od/id/mds2-2285. Accessed: 2020-9-10. 8

[4] Nist trojai challenge round3. https://data.nist. gov/od/id/mds2-2320. Accessed: 2020-11-7. 4, 8

[5] Naveed Akhtar, Mohammad AAK Jalwana, Mohammed Bennamoun, and Ajmal Mian. Label universal targeted attack. arXiv preprint arXiv:1905.11544, 2019. 2

[6] Eugene Bagdasaryan, Andreas Veit, Yiqing Hua, Deborah Estrin, and Vitaly Shmatikov. How to backdoor federated learning. arXiv preprint arXiv:1807.00459, 2018. 1

[7] Yang Bai, Yuyuan Zeng, Yong Jiang, Yisen Wang, Shu-Tao $\mathrm{Xia}$, and Weiwei Guo. Improving query efficiency of blackbox adversarial attack. arXiv preprint arXiv:2009.11508, 2020. 2

[8] Bryant Chen, Wilka Carvalho, Nathalie Baracaldo, Heiko Ludwig, Benjamin Edwards, Taesung Lee, Ian Molloy, and Biplav Srivastava. Detecting backdoor attacks on deep neural networks by activation clustering. arXiv preprint arXiv:1811.03728, 2018. 2, 4

[9] Huili Chen, Cheng Fu, Jishen Zhao, and Farinaz Koushanfar. Deepinspect: A black-box trojan detection and mitigation framework for deep neural networks. In Proceedings of the 28th International Joint Conference on Artificial Intelligence. AAAI Press, pages 4658-4664, 2019. 2

[10] Xinyun Chen, Chang Liu, Bo Li, Kimberly Lu, and Dawn Song. Targeted backdoor attacks on deep learning systems using data poisoning. arXiv preprint arXiv:1712.05526, 2017. 1, 2, 3

[11] Shuyu Cheng, Yinpeng Dong, Tianyu Pang, Hang Su, and Jun Zhu. Improving black-box adversarial attacks with a transfer-based prior. In Advances in Neural Information Processing Systems, pages 10932-10942, 2019. 2

[12] Edward Chou, Florian Tramèr, Giancarlo Pellegrino, and Dan Boneh. Sentinet: Detecting physical attacks against deep learning systems. arXiv preprint arXiv:1812.00292, 2018. 2
[13] Corinna Cortes and Vladimir Vapnik. Support-vector networks. Machine learning, 20(3):273-297, 1995. 5

[14] Kevin Eykholt, Ivan Evtimov, Earlence Fernandes, Bo Li, Amir Rahmati, Chaowei Xiao, Atul Prakash, Tadayoshi Kohno, and Dawn Song. Robust physical-world attacks on deep learning visual classification. In Proceedings of the IEEE Conference on Computer Vision and Pattern Recognition, pages 1625-1634, 2018. 3

[15] Clement Farabet, Camille Couprie, Laurent Najman, and Yann LeCun. Learning hierarchical features for scene labeling. IEEE transactions on pattern analysis and machine intelligence, 35(8):1915-1929, 2012. 1

[16] Yansong Gao, Chang Xu, Derui Wang, Shiping Chen, Damith C Ranasinghe, and Surya Nepal. Strip: A defence against trojan attacks on deep neural networks. arXiv preprint arXiv:1902.06531, 2019. 2, 8

[17] Ian J Goodfellow, Jonathon Shlens, and Christian Szegedy. Explaining and harnessing adversarial examples. arXiv preprint arXiv:1412.6572, 2014. 2

[18] Tianyu Gu, Brendan Dolan-Gavitt, and Siddharth Garg. Badnets: Identifying vulnerabilities in the machine learning model supply chain. arXiv preprint arXiv:1708.06733, 2017. 1,2

[19] Chuan Guo, Ruihan Wu, and Kilian Q Weinberger. Trojannet: Embedding hidden trojan horse models in neural networks. arXiv preprint arXiv:2002.10078, 2020. 2

[20] Wenbo Guo, Lun Wang, Xinyu Xing, Min Du, and Dawn Song. Tabor: A highly accurate approach to inspecting and restoring trojan backdoors in ai systems. arXiv preprint arXiv:1908.01763, 2019. 1, 2, 3

[21] Kaiming He, Xiangyu Zhang, Shaoqing Ren, and Jian Sun. Deep residual learning for image recognition. In Proceedings of the IEEE conference on computer vision and pattern recognition, pages 770-778, 2016. 4

[22] Geoffrey Hinton, Li Deng, Dong Yu, George E Dahl, Abdelrahman Mohamed, Navdeep Jaitly, Andrew Senior, Vincent Vanhoucke, Patrick Nguyen, Tara N Sainath, et al. Deep neural networks for acoustic modeling in speech recognition: The shared views of four research groups. IEEE Signal processing magazine, 29(6):82-97, 2012. 1

[23] Gao Huang, Zhuang Liu, Laurens Van Der Maaten, and Kilian Q Weinberger. Densely connected convolutional networks. In Proceedings of the IEEE conference on computer vision and pattern recognition, pages 4700-4708, 2017. 4

[24] Shanjiaoyang Huang, Weiqi Peng, Zhiwei Jia, and Zhuowen Tu. One-pixel signature: Characterizing cnn models for backdoor detection. arXiv preprint arXiv:2008.07711, 2020. 2

[25] Xijie Huang, Moustafa Alzantot, and Mani Srivastava. Neuroninspect: Detecting backdoors in neural networks via output explanations. arXiv preprint arXiv:1911.07399, 2019. 2

[26] Yujie Ji, Xinyang Zhang, Shouling Ji, Xiapu Luo, and Ting Wang. Model-reuse attacks on deep learning systems. In Proceedings of the 2018 ACM SIGSAC Conference on Computer and Communications Security, pages 349-363, 2018. 1 
[27] Soheil Kolouri, Aniruddha Saha, Hamed Pirsiavash, and Heiko Hoffmann. Universal litmus patterns: Revealing backdoor attacks in cnns. In Proceedings of the IEEE/CVF Conference on Computer Vision and Pattern Recognition, pages 301-310, 2020. 2, 5, 8

[28] Alex Krizhevsky, Vinod Nair, and Geoffrey Hinton. Cifar-10 (canadian institute for advanced research). 4

[29] Alex Krizhevsky, Ilya Sutskever, and Geoffrey E Hinton. Imagenet classification with deep convolutional neural networks. In Advances in neural information processing systems, pages 1097-1105, 2012. 1

[30] Alexey Kurakin, Ian Goodfellow, and Samy Bengio. Adversarial examples in the physical world. arXiv preprint arXiv:1607.02533, 2016. 2

[31] Yann LeCun and Corinna Cortes. MNIST handwritten digit database. 2010. 4

[32] Huichen Li, Xiaojun Xu, Xiaolu Zhang, Shuang Yang, and Bo Li. Qeba: Query-efficient boundary-based blackbox attack. In Proceedings of the IEEE/CVF Conference on Computer Vision and Pattern Recognition, pages 1221-1230, 2020. 2

[33] Maosen Li, Cheng Deng, Tengjiao Li, Junchi Yan, Xinbo Gao, and Heng Huang. Towards transferable targeted attack. In Proceedings of the IEEE/CVF Conference on Computer Vision and Pattern Recognition, pages 641-649, 2020. 2

[34] Kang Liu, Brendan Dolan-Gavitt, and Siddharth Garg. Finepruning: Defending against backdooring attacks on deep neural networks. In International Symposium on Research in Attacks, Intrusions, and Defenses, pages 273-294. Springer, 2018. 2

[35] Yingqi Liu, Wen-Chuan Lee, Guanhong Tao, Shiqing Ma, Yousra Aafer, and Xiangyu Zhang. Abs: Scanning neural networks for back-doors by artificial brain stimulation. In Proceedings of the 2019 ACM SIGSAC Conference on Computer and Communications Security, pages 1265-1282, 2019. 2

[36] Yingqi Liu, Shiqing Ma, Yousra Aafer, Wen-Chuan Lee, Juan Zhai, Weihang Wang, and Xiangyu Zhang. Trojaning attack on neural networks. 2017. 1, 2

[37] Yunfei Liu, Xingjun Ma, James Bailey, and Feng Lu. Reflection backdoor: A natural backdoor attack on deep neural networks. arXiv preprint arXiv:2007.02343, 2020. 2

[38] Aleksander Madry, Aleksandar Makelov, Ludwig Schmidt, Dimitris Tsipras, and Adrian Vladu. Towards deep learning models resistant to adversarial attacks. arXiv preprint arXiv:1706.06083, 2017. 2

[39] Tomáš Mikolov, Anoop Deoras, Daniel Povey, Lukáš Burget, and Jan Černockỳ. Strategies for training large scale neural network language models. In 2011 IEEE Workshop on Automatic Speech Recognition \& Understanding, pages 196-201. IEEE, 2011. 1

[40] Seyed-Mohsen Moosavi-Dezfooli, Alhussein Fawzi, Omar Fawzi, and Pascal Frossard. Universal adversarial perturbations. In Proceedings of the IEEE conference on computer vision and pattern recognition, pages 1765-1773, 2017. 7

[41] Seyed-Mohsen Moosavi-Dezfooli, Alhussein Fawzi, and Pascal Frossard. Deepfool: a simple and accurate method to fool deep neural networks. In Proceedings of the IEEE conference on computer vision and pattern recognition, pages 2574-2582, 2016. 2, 5

[42] Genki Osada, Budrul Ahsan, Revoti Prasad Bora, and Takashi Nishide. Regularization with latent space virtual adversarial training. In European Conference on Computer Vision, pages 565-581. Springer, 2020. 2

[43] Nicolas Papernot, Patrick McDaniel, Ian Goodfellow, Somesh Jha, Z Berkay Celik, and Ananthram Swami. Practical black-box attacks against machine learning. In Proceedings of the 2017 ACM on Asia conference on computer and communications security, pages 506-519, 2017. 3

[44] Ximing Qiao, Yukun Yang, and Hai Li. Defending neural backdoors via generative distribution modeling. In Advances in Neural Information Processing Systems, pages 1400414013, 2019. 1, 2

[45] Adnan Siraj Rakin, Zhezhi He, and Deliang Fan. Tbt: Targeted neural network attack with bit trojan. In Proceedings of the IEEE/CVF Conference on Computer Vision and Pattern Recognition, pages 13198-13207, 2020. 2

[46] Aniruddha Saha, Akshayvarun Subramanya, and Hamed Pirsiavash. Hidden trigger backdoor attacks. In Proceedings of the AAAI Conference on Artificial Intelligence, volume 34, pages 11957-11965, 2020. 2

[47] Karen Simonyan and Andrew Zisserman. Very deep convolutional networks for large-scale image recognition. arXiv preprint arXiv:1409.1556, 2014. 4

[48] Christian Szegedy, Wei Liu, Yangqing Jia, Pierre Sermanet, Scott Reed, Dragomir Anguelov, Dumitru Erhan, Vincent Vanhoucke, and Andrew Rabinovich. Going deeper with convolutions. In Proceedings of the IEEE conference on computer vision and pattern recognition, pages 1-9, 2015. 4

[49] Christian Szegedy, Wojciech Zaremba, Ilya Sutskever, Joan Bruna, Dumitru Erhan, Ian Goodfellow, and Rob Fergus. Intriguing properties of neural networks. arXiv preprint arXiv:1312.6199, 2013. 2

[50] Tuan A Tang, Lotfi Mhamdi, Des McLernon, Syed Ali Raza Zaidi, and Mounir Ghogho. Deep learning approach for network intrusion detection in software defined networking. In 2016 International Conference on Wireless Networks and Mobile Communications (WINCOM), pages 258-263. IEEE, 2016. 1

[51] Jonathan J Tompson, Arjun Jain, Yann LeCun, and Christoph Bregler. Joint training of a convolutional network and a graphical model for human pose estimation. In Advances in neural information processing systems, pages 1799-1807, 2014. 1

[52] Florian Tramèr, Alexey Kurakin, Nicolas Papernot, Ian Goodfellow, Dan Boneh, and Patrick McDaniel. Ensemble adversarial training: Attacks and defenses. arXiv preprint arXiv:1705.07204, 2017. 2

[53] Brandon Tran, Jerry Li, and Aleksander Madry. Spectral signatures in backdoor attacks. In Advances in Neural Information Processing Systems, pages 8000-8010, 2018. 1, 2

[54] BS Vivek and R Venkatesh Babu. Single-step adversarial training with dropout scheduling. arXiv, pages arXiv-2004, 2020. 2 
[55] Weitao Wan, Jiansheng Chen, and Ming-Hsuan Yang. Adversarial training with bi-directional likelihood regularization for visual classification. 2

[56] Bolun Wang, Yuanshun Yao, Shawn Shan, Huiying Li, Bimal Viswanath, Haitao Zheng, and Ben Y Zhao. Neural cleanse: Identifying and mitigating backdoor attacks in neural networks. Neural Cleanse: Identifying and Mitigating Backdoor Attacks in Neural Networks, page 0, 2019. 1, 2, 8

[57] Qinglong Wang, Wenbo Guo, Kaixuan Zhang, Alexander G Ororbia, Xinyu Xing, Xue Liu, and C Lee Giles. Adversary resistant deep neural networks with an application to malware detection. In Proceedings of the 23rd ACM SIGKDD International Conference on Knowledge Discovery and Data Mining, pages 1145-1153, 2017. 1

[58] Ren Wang, Gaoyuan Zhang, Sijia Liu, Pin-Yu Chen, Jinjun Xiong, and Meng Wang. Practical detection of trojan neural networks: Data-limited and data-free cases. arXiv preprint arXiv:2007.15802, 2020. 2

[59] Zhen Xiang, David J Miller, and George Kesidis. Revealing backdoors, post-training, in dnn classifiers via novel inference on optimized perturbations inducing group misclassification. In ICASSP 2020-2020 IEEE International Conference on Acoustics, Speech and Signal Processing (ICASSP), pages 3827-3831. IEEE, 2020. 2

[60] Chang Xiao and Changxi Zheng. One man's trash is another man's treasure: Resisting adversarial examples by adversarial examples. In Proceedings of the IEEE/CVF Conference on Computer Vision and Pattern Recognition, pages 412-421, 2020. 2

[61] Han Xiao, Kashif Rasul, and Roland Vollgraf. Fashionmnist: a novel image dataset for benchmarking machine learning algorithms. arXiv preprint arXiv:1708.07747, 2017. 4

[62] Chulin Xie, Keli Huang, Pin-Yu Chen, and Bo Li. Dba: Distributed backdoor attacks against federated learning. In International Conference on Learning Representations, 2019. 2

[63] Xiaojun Xu, Qi Wang, Huichen Li, Nikita Borisov, Carl A Gunter, and Bo Li. Detecting ai trojans using meta neural analysis. arXiv preprint arXiv:1910.03137, 2019. 2, 8

[64] Yuanshun Yao, Huiying Li, Haitao Zheng, and Ben Y Zhao. Latent backdoor attacks on deep neural networks. In Proceedings of the 2019 ACM SIGSAC Conference on Computer and Communications Security, pages 2041-2055, 2019. 2

[65] Dinghuai Zhang, Tianyuan Zhang, Yiping Lu, Zhanxing Zhu, and Bin Dong. You only propagate once: Accelerating adversarial training via maximal principle. In Advances in Neural Information Processing Systems, pages 227-238, 2019. 2

[66] Zaixi Zhang, Jinyuan Jia, Binghui Wang, and Neil Zhenqiang Gong. Backdoor attacks to graph neural networks. arXiv preprint arXiv:2006.11165, 2020. 2

[67] Shihao Zhao, Xingjun Ma, Xiang Zheng, James Bailey, Jingjing Chen, and Yu-Gang Jiang. Clean-label backdoor attacks on video recognition models. In Proceedings of the IEEE/CVF Conference on Computer Vision and Pattern Recognition, pages 14443-14452, 2020. 2
[68] Minhui Zou, Yang Shi, Chengliang Wang, Fangyu Li, WenZhan Song, and Yu Wang. Potrojan: powerful neurallevel trojan designs in deep learning models. arXiv preprint arXiv:1802.03043, 2018. 1 